\title{
Development of Indoor Spatial Data Model Using CityGML ADE
}

\author{
Yun-Ji Kim ${ }^{\mathrm{a}}$, Hye-Young Kang ${ }^{\mathrm{b}}$, Jiyeong Lee \\ ${ }^{\text {abc }}$ Dept. Of Geoinformatics, University of Seoul \\ hoqpdlq1@uos.ac.kra ,hyezero@gmail.com ${ }^{\mathrm{b}}$, jlee@uos.ac.kr
}

\begin{abstract}
With the recent increasing build and application for 3D spatial information, the importance of management and application for spatial information based on indoor space has been increased. Especially, Due to the increasing of the scale and complexity of the building according to the development of construction technologies several studies have been conducted to provide the services based on indoor space such as indoor navigation for disaster. Therefore, to efficient manage and service for information of complicated indoor space, it is necessary to extend and develop 3D spatial model and services that have been developed for outdoor space. In this paper, Indoor Spatial Data Model (ISDM) is developed to support building spatial information for complicated indoor space and location based services through topological information. ISDM contains a feature model which is a CityGML Application Domain Extension (ADE) model and a topology model that refers the IndoorGML
\end{abstract}

Key Word: Indoor Spatial Data Model, 3D Data Model, CityGML

\section{INTRODUCTION}

With the recent interests in 3D spatial information used for location based services, various 3D urban models used in many areas such as urban planning, disaster management have been developed. Especially, after 9/11 and 7/7, the need of building spatial information for not only outdoor spaces but also indoor spaces have grown rapidly. Due to increasing of the size and complexity of the building several studies have been conducted to provide the services based on indoor space $[5,8,11]$. To manage and utilize efficiently for complex indoor space, data model which can be utilized and various services needs to develop by extending from 3D spatial information and service that have mainly developed outdoor space to indoor space. In particular, the most important and basic thing is how to develop and implement 3D data model to provide 3D spatial information and location based services of indoor space. Existing spatial information and application services have mostly developed targeting outdoor space, and data models focusing on outdoor space also have been defined and improved.

Recent efforts have proposed several spatial data models in 3D space. CityGML [10] is a data format for exchange and storage of virtual 3D city model, which is based on XML data model to representative open data model. Purpose of developing CityGML is intended to define common entity, attribute, and relation of 3D city model that can be shared among other applications [8]. CityGML basically refers to
ISO 19100 series and the other documents about XML standards of W3C. CityGML consists of Geometry model and thematic model. Geometry model defines geographical and topological attributes of spatial objects in 3D city model as in figure 1 [10]. CityGML can be used to represent indoor space, but it cannot represent topological relationships among objects of the indoor space. IFC (Industry Foundation Classes) has been adopted BIM data model by applying the object-oriented method and adopted XML as a method of expression [1]. This standard data model was created to support interoperability among architecture, engineering and AEC and registered in the ISO/PAS16739 [4]. IFC has defined data elements indicating the composition of the spatial relationships of a building and other buildings and included information related to spatial extents of the composition. IFC has the advantages describing object of inside building in detail. However, it is not able to represent the topological information because only simple objects have been represented. In the previous researches, they are focused on representing the geometry of objects. It can be used as a visualization model, but has limitations for spatial analysis.

Therefore, this study proposes Indoor Spatial Data Model (ISDM) representing the geometry and the topology of indoor space developed to support indoor location based services. 


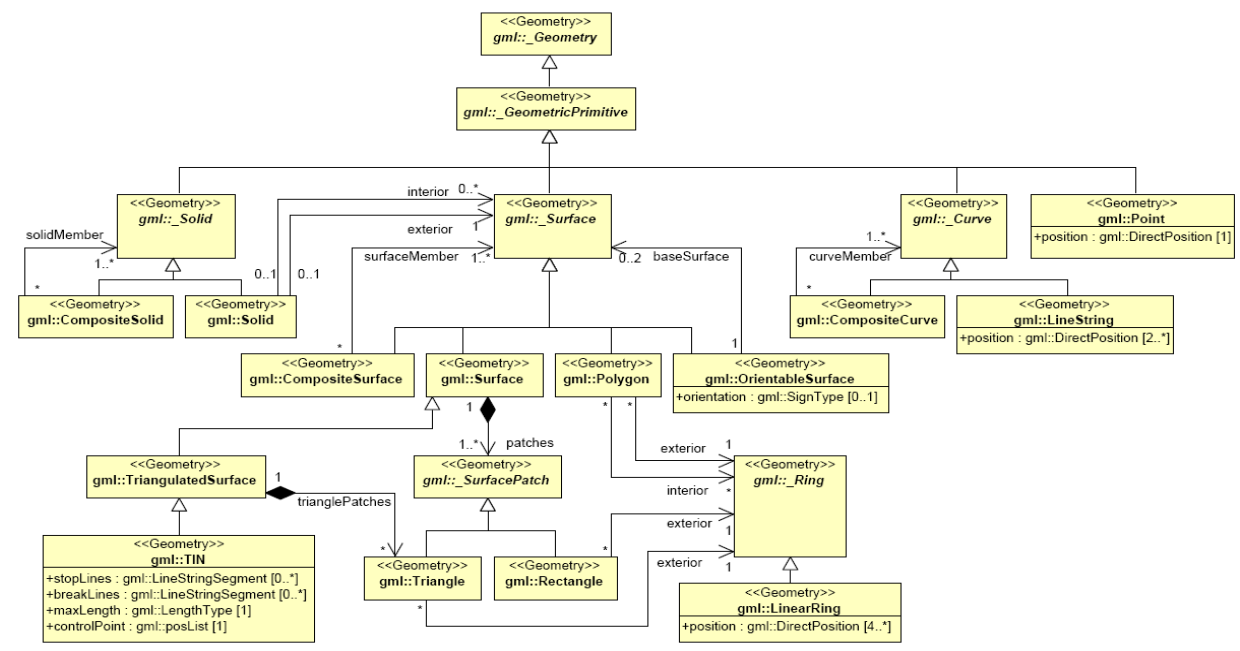

Figure1. UML diagram of CityGML geometry model (Primitives and Composites) [5]

\section{Indoor Spatial Data Model}

ISDM (Indoor Spatial Data Model) is proposed to provide 3D indoor location based services. The ISDM has been developed based on concepts which are CityGML ADE [10] and IndoorGML [9]. CityGML ADE (Application Domain Extensions) is an extended model for CityGML and IndoorGML is a topological model, which is under developing in OGC IndoorGML SWG.

This study proposes Indoor Spatial Data Model considering international standards and compatibility. CityGML ADE is a model that has been appropriately extended to represent indoor space to further define required objects and the relationships to illustrate indoor space in City Object, Building, and Transportation Module of CityGML. Figure 2 shows a conceptual diagram of Indoor Spatial Data Model developed in this study.

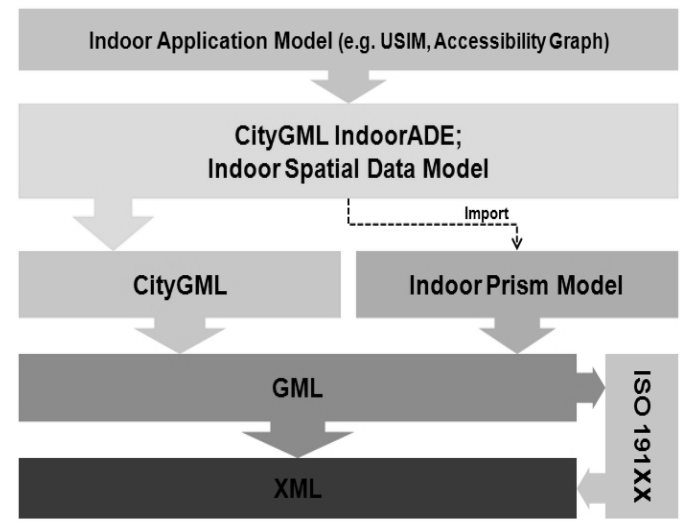

Figure 2. Basic concept of development for Indoor Spatial Data Model

Indoor Spatial Data Model supports spatial analysis based on location. For this reason, NRS (Node-Relation-Structure) [7] of network architecture concept has been used to represent topological relationships such as connectivity and adjacency. NRS can make complex topologic representations simple and abstract among 3D unit spaces of indoor environment such as rooms in the building. NRS utilizes Poincare Duality to simplify complex spatial relationship among 3D objects by topology network model. In figure 3, $3 \mathrm{D}$ objects are represented by nodes and relationships between 3D objects are described by edges. Concept of NRS has been used in Indoor GML.

ISDM has two models; Core Indoor Model and Building Indoor Model, and Core Indoor Model will be introduced first.

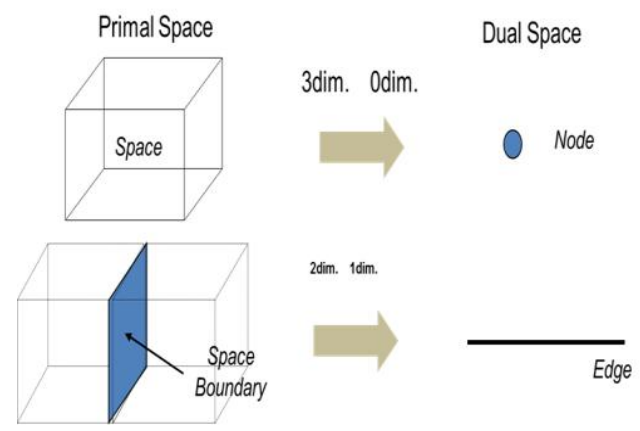

Figure 3. Basic concept of development for Indoor Spatial Data Model [7]

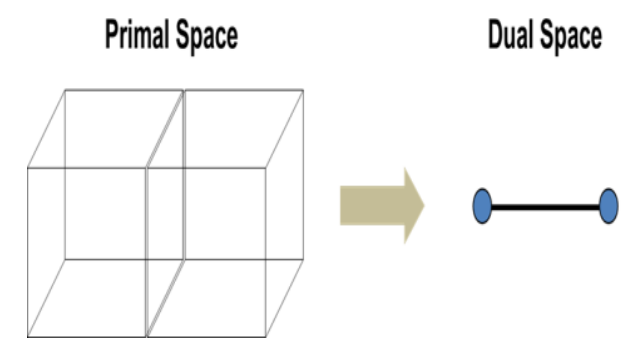

Figure 4. Poincare duality of 3D objects and relationship between $3 \mathrm{D}$ objects [7]

\subsection{Core Indoor Model}

Core Indoor Model of ISDM is a module defining superclasses to describe 3D indoor space. Core module is defined to be represented indoor space with reference to theme modules and Core Module of CityGML. In figure 5, 
UML diagram of proposed model in this study can be seen. GML class includes gml::Feature and gml::FeatureCollection, CityGML core module class includes CityModel, CityObject, and_Site, and CityGML thematic extension module class includes app::Appearance, $\quad$ tran::_TransportationObject, frn::CityFurniture, bldg::_AbstractBuilding. CityGML Indoor ADE class has been newly added. It represents Indoor::MovingObject, which include MovingObjectID,
MovingObjectName,

MovingObjectProperty, MovingObjectLocation, MovingObjectVelocity, and MovingObjectTrajectory. CityModel has CityObject and app::Appearance using shared aggregation, and CityObject also has app::Appearance using shared aggregation. gml::Feature is implemented by CityObject, gml::FeatureCollection is implemented by CityModel implemented by _site, tran::_TransportationObject, frn::CityFurniture, and Indoor::MovingObject.

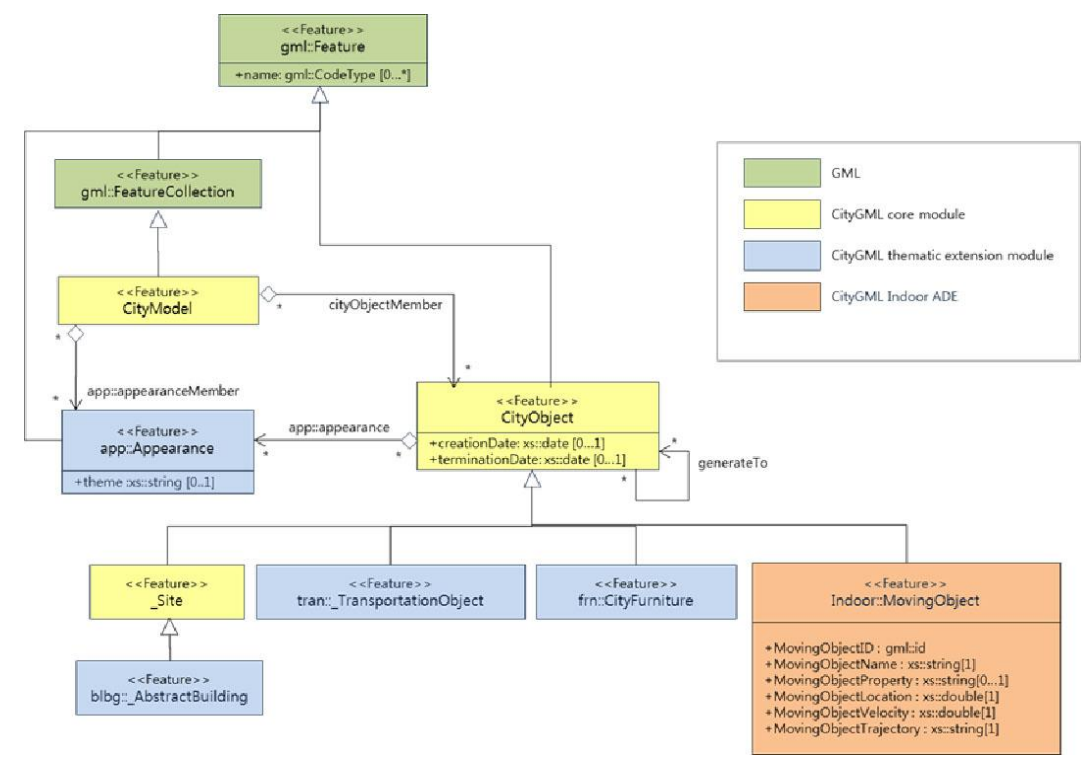

Figure 5. Core Indoor model of ISDM

\subsection{Building Indoor Model}

As the second Model of ISDM, Building Indoor Model represents required elements to describe the building, as well the basis of the building module of CityGML. ExteriorBuildingObject and InteriorBuildingObject are added to distinguish between the external space and the internal space of the building, and added StoreyObject represents the floor unit of the building. This is illustrated interior building part in figure 6. ExteriorBuildingObject is a feature class defined in inheritance from
Core::CityObject of CityGML and has aggregation of _AbstractBuilding and IntBuildingInstallation which can define attributes and characteristics with reference to IndoorGML::SpaceObject defined by IndoorGML. The geometry of class which can be represented spatially is expressed by reference to IndoorPrism::_Solid, and BoundarySurface refers to IndoorGML::AbstractSpaceBoundary to use in spatial analysis. Indoor::Storey consists of a set of InteriorBuldingObject and represents each floor of the building. Indoor::Storey has 'ID' to distinguish the floor and 'name' to describe name of the floor as an attribute. 


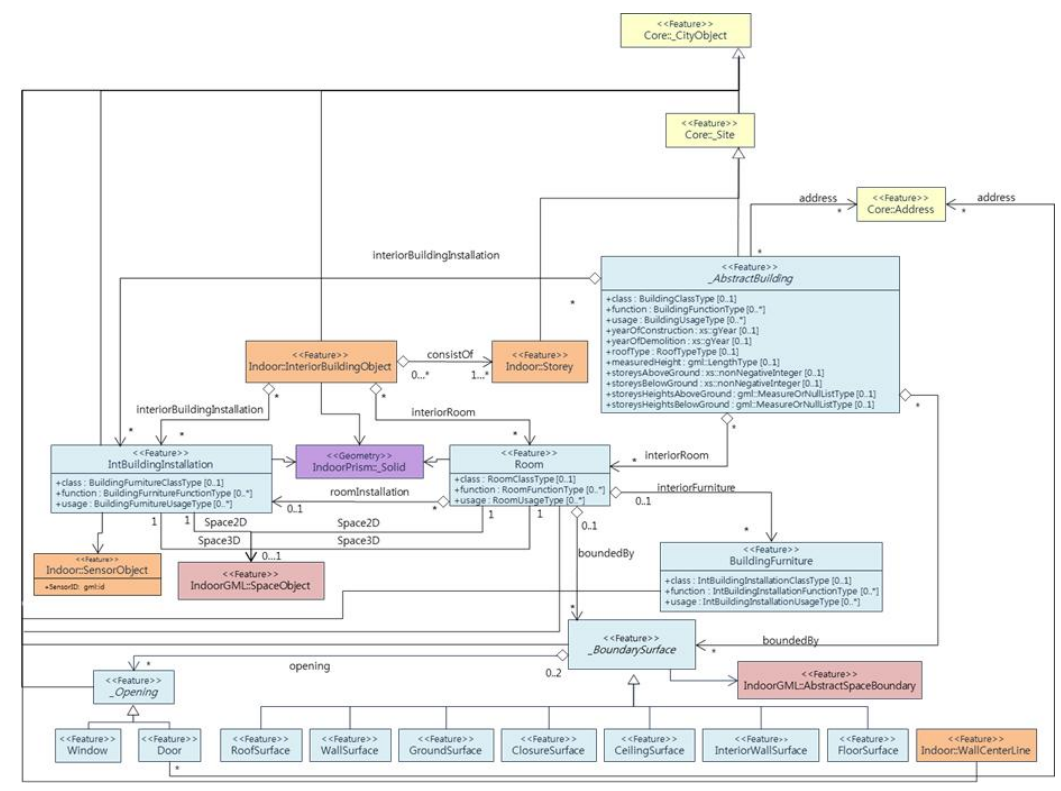

Figure 6. Indoor Building Part

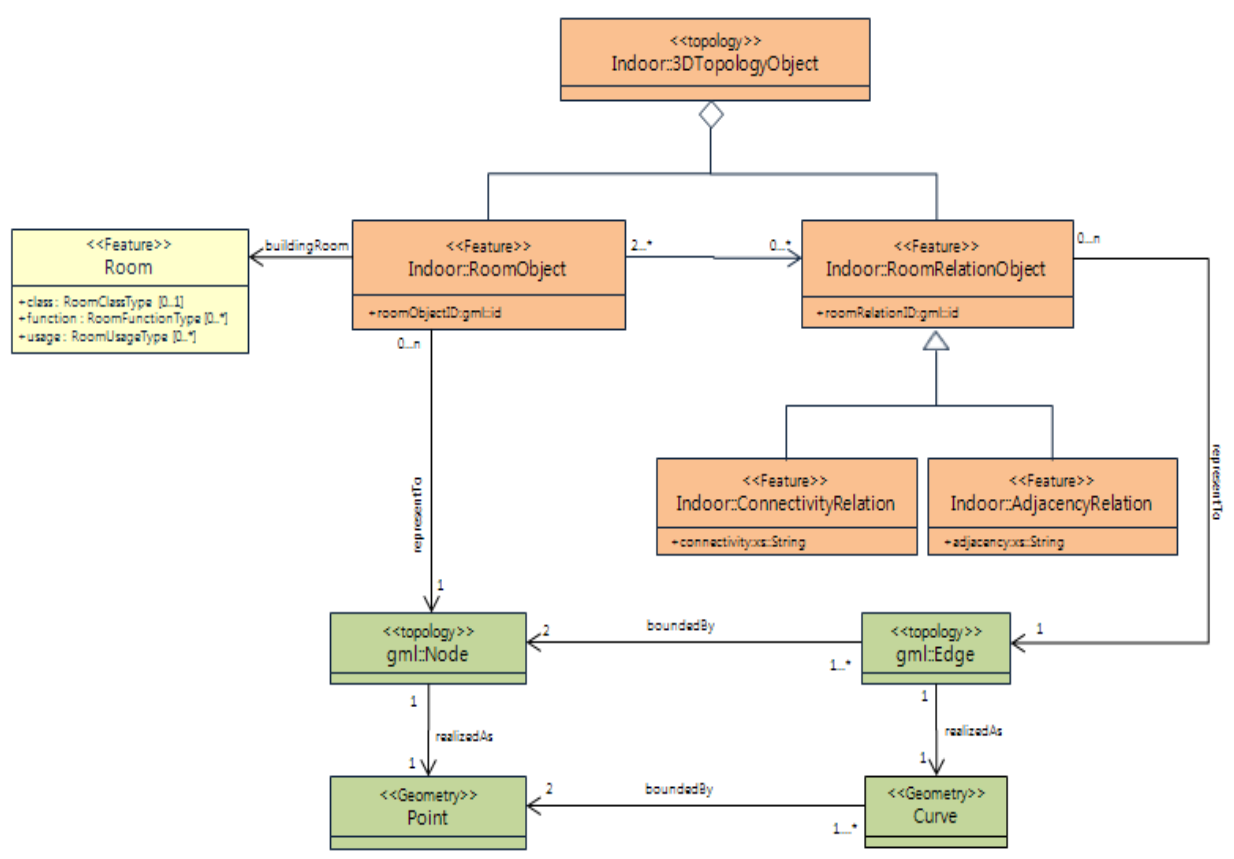

Figure 7. 3D Object Topology Model, CityGML Indoor ADE

\subsection{D Object Topology Model}

The last proposed module in this study is the 3D Object Model representing the relationship of 3D objects in the space. 3DTopologyObject is a super class, and it has RoomObject, RoomRelationObject as subclasses and Generic Attribute. RoomObject which is represented by a node in Topology Primitive refers to Room class depicting indoor space of building as three-dimensions. Each spaces represented by $3 \mathrm{D}$ has a relationship between the respective spaces, and these relationships can be shown as a face. Relation of spatial objects expressed by the RoomRelationObject class leads to edge in ISDM and in that spatial relationships represented by a face can lead to edge.
RoomRelationObject has ConnectivityRelation defined in relation with the continuity and AdjacencyRelation defined in relation with the adjacency as subclasses. 3D Object Topology Model is shown as a figure 7.

\section{IMPLEMENTATION AND VISUALIZATION}

The proposed ISDM was implemented by generating and visualizing an XML data document based on the XML schema. Study area is the $21^{\text {st }}$ Century Building, in University of Seoul, South Korea. The XML data was made on the basis of the study area and the ISDM Viewer was developed to visualize XML Data Documents. The figure 8 shows the sample of XML data describing indoor space. 3D 
indoor spatial data based on XML schema of ISDM describes relationship clearly among spatial objects as well as geometric representation of real 3D indoor space.

We have developed a viewer for visualization for verification of error in the data based on XML schema of ISDM. When the viewer is opened by XML data of the building, it will show visualized spatial data such as figure 9 .

It is possible to visually confirm not only the geometric representation of ISDM, but also the representation of the relationships of spaces using the viewer.

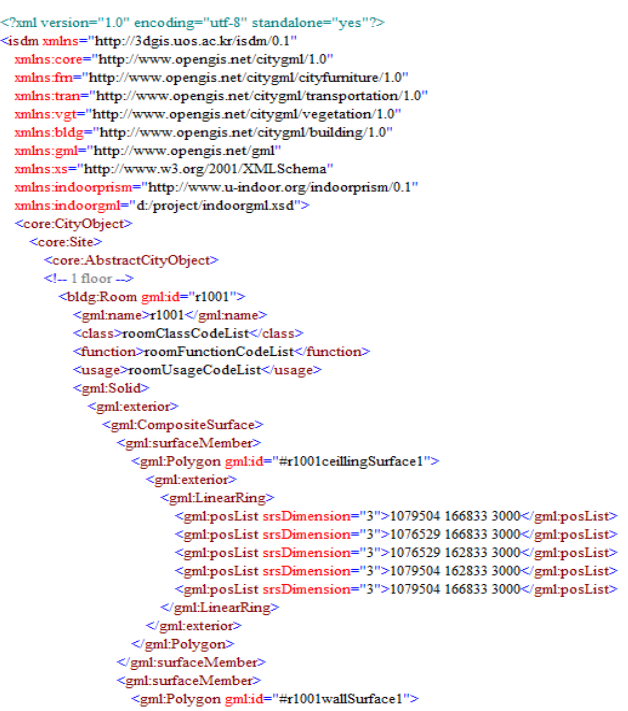

Figure 8. A sample XML data

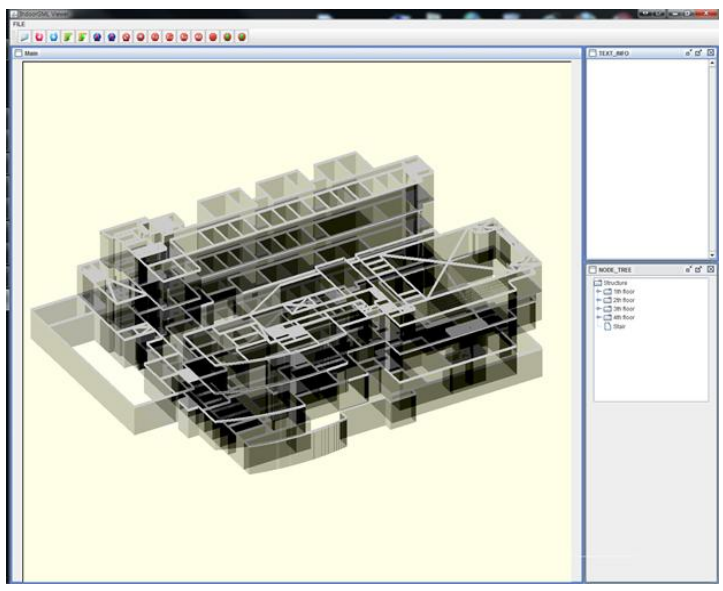

Figure 9. ISDM Viewer: Sample data

\section{CONCLUSION}

While the recent increasing build and application for 3D spatial information, the importance of management and application for spatial information based on indoor space has been increased. CityGML which is possible to depict outward expression simply includes extended model to describe indoor space among developed various 3D urban models, but it is not enough to support the representation and service about indoor space. Therefore, this study proposes ISDM of application domain extension based on CityGML. ISMD has defined additional feature classes to represent indoor space based on the extended modules of CityGML. Further, topological model of $3 \mathrm{D}$ indoor space is defined with reference to IndoorGML and XML schema is constructed on basis of the definition. Designed XML schema in this paper could represent geometric expression of indoor space and topologic representation which makes spatial analysis possible.

\section{ACKNOWLEDGEMENT}

This research was supported by a grant(11 High-tech Urban G11) from Architecture \& Urban Development Research Program and by the supporting project to educate GIS experts funded by Ministry of Land, Infrastructure and Transport of Korean government.

\section{REFERENCES}

1. IAI(International Alliance for Interoperability), www.iaiinternational.org.

2. ISO 10303-11:2004, Industrial automation systems and integration - product data representation and exchange Part 11: Description methods: The EXPRESS language reference manual.

3. ISO 19107:2003, Geographic information - Spatial schema.

4. ISO/PAS 16379:2005, Industry Foundation Classes, Release 2x, Platform Specification.

5. Kwak, S. Y; Nam, H. W; Jun, C. M. 2010, An Indoor Pedestrian Simulation Model Incorporating the Visibility, Journal of Korea Spatial Information Society, 18(5):133142.

6. Kim, J. S; Kang, H. Y; Lee, T. H; Li, K. J. 2009, Topology of the Prism Model for 3D Indoor Spatial Objects, Proc. International Workshop on Indoor Spatial Awareness, pp.698-703.

7. Lee, J. Y. 2004, A Spatial Access Oriented Implementation of a Topological Data Model for 3D Urban Entities, GeoInformatica, 8(3):235-262.

8. Lee, S. J; Lee, J. Y. 2011, Navigable Space-Relation Model for Indoor Space Analysis, Journal of Geographic Information System Association of Korea, 19(5):75-86

9. Nagel, C.; Becker B.; Kaden, R.; Li,K-J.;Lee, J.; and Kolbe, T. H. 2010, Requirements and Space-Event Modeling for Indoor Navigation, OGC 10-191rl.

10. OGC. 2008, City Geography Markup Language (CityGML) Encoding Standard, OGC 08-001rl.

11. Park, I. H; Jun, C. M; Choi, Y. S. 2007, 3D-GIS Network Modeling for Optimal Path Finding in Indoor Space, Journal of the Korea Society for GeoSpatial Information System, 15(3):27-32. 